\title{
Ontology Based Simulation Framework: Studying of Human Behavior Changes Impacted by Accessibility of Information under Building Fire Emergency
}

\author{
Chaianun Damrongrat and Mitsuru Ikeda
}

School of Knowledge Science

Japan Advanced Institute of Science and Technology, Ishikawa, Japan 923-1211

\{chaianun.d, ikeda\}@jaist.ac.jp

http://www.springer.com/lncs

\begin{abstract}
In gerneal, human behavior under emergency situation is considered as irrational behavior. However, recent research studies showed that evacuees had rational decision making process even though they said they were panic at that time. Evacuees behaved rationally based on information they could access at that moment and then selected the best option for response. This research is interested in relationship between human behavior and accessibility of informaiton under the restricted information situation, emergency in this case. We propose an ontologybased simulation framework as a tool to find out the relationship. Even though it is hard to claim that human will behave as same as the simulation outcome, we could study the relationship by observing trend and tendency of human behavior changes in the simulation by varying simulation parameters. For this purpose, the simulation models should be easy to modify. Then ontology plays a key component for this issue.
\end{abstract}

Keywords: ontology based simulation, human behavior, accessibility of information, emergency situation, restricted information.

\section{Introduction}

In restricted information situation, such as a building fire emergency, there were many people injured and died because they did not know and/or overlooked vital information [1, 2]. In this such situation, people should evacuate to safe places as fast as they could. However, due to the lack of accessible information, incorrect information and time limit, this situation is hard for evacuees to make a right decision to respond the situation effectively.

Research studies showed that occupants behave rationally under emergency. Some behaviors might look irrationally and hesitantly but that was because they were lack of important information in their hand. It made they had a hard time to make a good decision. Research experiment showed that ones who did not know where the emergency exits were will escape to the entrance they entered 
even though there was a nearer emergency exit in the area. On the other hand, ones who know the information of emergency exits selected to escape by the nearest exit [2, 3. This showed that occupants responded differently if they have different accessibility of information.

This research interested to know how accessibility of information can make an impact to human behavior. We have a hypothesis following existing findings that occupants have rational behavior under the critical situation [4, 5, 6]. They react based on the information they have at that time. In another word, their behavior is based on accessibility of information. To extend the existing findings, we aims to find hidden relationship among human behaviors changes and accessibility of information under restricted information situation. This research proposes an ontology-based simulation framework to find the relationship between human behavior and accessibility of information under restricted-information situation. We use an indoor building fire emergency as an example for our research domain. Even though we could not claim that human behavior will react exactly the same as simulation outcome; however, we could find trend or tendency of human behavior changes by varying simulation parameters. Since the research study is rely on tendency of the simulation outcome, it is better to have an approach that flexible enough in modifying the behavior models. Then the ontology is used in this research describing models of human behaviors and environment's character such as fire development. The ontology is mainly used for three purposes. The first is a tool to transfer knowledge from domain experts such as interviewing of fire fighters or safety guidelines to become machine-readable data which is used in simulation system. The second is using ontology reasoning to find the hidden relation among human behavior and accessibility of information. The last one is flexibility of reusing and sharing knowledge. We can edit or change situation model or behavior model easily with new ontology. Moreover, this ontology-based simulation framework could be used as a tool to verify hypotheses of finding relationship among an accessibility of information, human behavior and critical situation in different interested problem.

Understanding this relationship could help us, researchers, to understand how to maximise benefit of accessibility of information effectively. For example, in a limited time and resources we cannot provide all information the sensors have to occupants. The knowing could point out what type of information is a vital information and when to provide that information to the right ones who need them to escape the emergency.

\section{Human Behavior under Emergency}

Human behavior under emergency, in general, is considered as irrational behavior because of panic. However, research studies showed that evacuees in an indoor emergency took action with rational behavior based on information they had at that time even though they said themselves they were panic at that moment [4], [5], 6]. 


\subsection{Behaviors under Overloading Information Situation and Restricted Information Situation}

Research studies on human behavior under overloading information situation mostly based on research of natural disasters. People tried to contact to others for informing and checking current situation from the both ends of communication [7] [8. This behavior might not so relate with this research because natural disaster and building fire have different characters. However, it could reflex some problem of human behavior under rich information situation. Another case study is overuse the alarms could make people immune and ignore them when the emergency is really happened [9]. This showed that individuals have behavior to ignore the information if they believe the information is abused. For restricted information situation, there are many research studies and reports showed that evacuees tried to escape the emergency by the main entrances or normally-used exits but overlooked the emergency exits which might be nearer ones [2], [3], 10].

\subsection{Human Behavior in Psychological Perspective}

Research studies showed that there are many behaviors the evacuees might express under the critical situation. For example, herding behavior and altruistic behavior. Herding behavior is often observed during an evacuation of a room, for example, with two exits. One exit is crowded with evacuees while another exit is not fully utilized. There were many event that herding behavior happened such as a fire in the Station nightclub, 211 Cowesett Anenue, West Warwick, Rhode Island which the evacuees tried to escape by the main entrance of the building and caused approximately one hundred people died [10]. Altruistic behavior is happened when the individual feel empathy to another. The individuals are more willing to help ones in need if they observed that others initiate help [1]. However, these behaviors require to observe other individuals' behavior. This research will focus on modeling an individual behavior, and will let the social behavior in the future work.

\section{Methodology}

To studying the human behavior changes impacted by accessibility of information, we propose a simulation framework which use ontology to enhance an ability of modifying the behavior models. To find out what are the behavior changes, we have to compare two simulation outcomes. These two simulations have to processed under the same controlled set of simulation parameters such as agents properties, decision making process, group size of agents and environment's properties. The only difference between the two simulations is level of information accessibility. One simulation has limited accessibility of information, another one has better level of information accessibility. 


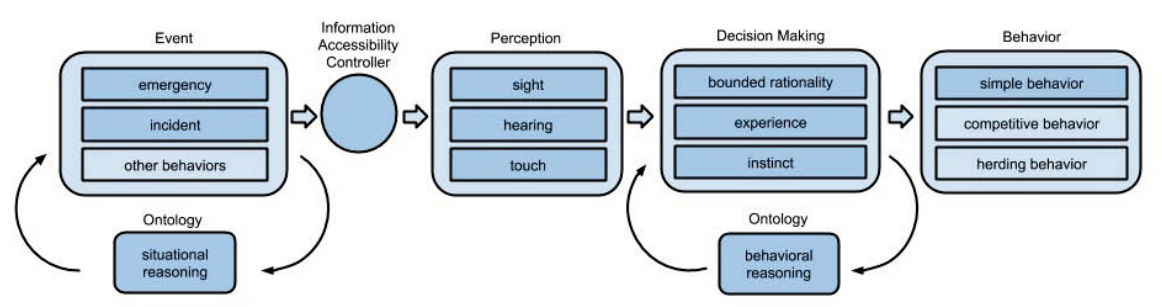

Fig. 1. In this simulation framework we focus on individual behavior only, as be showed with dark blue color. Behavior interacting with social such as competitive behavior or herding behavior are not include in this research study yet, as be showed with light blue color. Ontology is used to model the emergency situation, simple behavior such as avoiding to be close to dangerous area, and model the consequence of emergency situation and human behavior by its reasoning. In this research we are interested in how information accessibility have impacted to human behavior. Then we have a module controlling level of information to human agents in simulation.

\subsection{Simulation Framework}

In studying relationship between human behavior and information accessibility, we have intension to propose a simulation framework which flexible enough to handle to evaluate new hypotheses. This means the framework should be comfort to modify modules such as behavior models. To achieve this purpose we embeded ontology to the existing study to utilize the advantages of ontology such as flexibility of modifying the human behavior models, reuse and reshare knowledge in the framework. The research study proposed a process model describing emergency behaviors has four major modules, cues or event, perception, decision making and behavior [12] as showed in Fig, 1. Without ontology module, the simulation framework would be highly depend on modelers or domain experts who normally might not familiar with IT such as simulation model. Ontology could be a tool to overcome this gap because it is a human-and-machine readable language. Since this research focus on study relationship between individual behavior and information accessibility. The social behaviors, as showed in light blue in Fig 1, will be omit at this time.

\subsection{Ontology}

This simulation framework uses ontology to model an agent behavior, relationship among agents, relationship between agent and environment. For example, an agent should avoid to go to dangerous areas. However, this research is an initial state of using this framework, the complex concept models are not applied to this work yet. In the near future we could utilize the benefit of using ontology to conceptualize knowledge to make more complex model. Moreover, ontology is good to separate domain knowledge from the operation knowledge [13. This means we can define our behavior model more complex and more realistic from 
assistance of domain experts such as fire fighters who may not well understanding how the simulation system works. Without ontology, the domain experts may have to understand how the system works while making a new model. It is not convenient for domain experts and take time.

Ontology Design. The research goal is to study the relationship among human behavior and information accessibility under a critical situation. However, this research considers on individual behavior. The interaction behavior among agents will be left to the future work. This section describe how the ontology is designed. The ontology is used to describe concepts of human behavior, information accessibility and critical situation, respectively. The overview ontology is showed in Fig 2 which some social behaviors in the fureture work are prepared. In this paper, the individual behavior is considered based on what behaviors that an agent can do in the simulation. The two main behaviors are Avoid and Escape. An agent should have behavior to avoid dangerous places, and should escape to safe places in evacuation. After that we consider what the information accessibility is. We make a Perception concept to describe how to access information. However, this research considers on the simple simulation. The information accessibility in this research is not complex yet. It is only on and off statement. For the critical situation, we have to define concepts of Dangerous and Safe to define dangerous place and safe place in the simulation. In this paper, we define fire as relating to danger. Any place with fire is a dangerous place, otherwise is safe place.

\subsection{Simulation Methods}

There are some research studies related to evacuation in emergency situation. Crowd behavior was proposed to represent the human behavior under emergency. Research studies compared human behavior with fluid and particle motion analogy 14. However, Later studies showed that fluid analogies of crowd were untenable 15. Herding behavior was proposed instead based on the observation that in a room with two exits, one exit was clogged while the other was not fully utilized [16. This behavior represents human behavior in real situation which the fluid analogy model could not explain this behavior properly. These research studies focus on social behavior. They required high computational power since the model had to concern about each agents movement in the simulation environment and interaction among agents. To avoid the high computation of agents' movement, another model such as matrix-based simulation was proposed [17. it discretized a floor area into cells. This approach was simple and easy to understand to model the environment scene; however, the model highly depended on the modelers skill. In our research we attempt to use simple part of a small unit, such as behavior of an individual agent, to explain complex phenomena. This research do not consider about the detail of how an agents movement is in the simulation environment. We consider the outcome of its action such as 


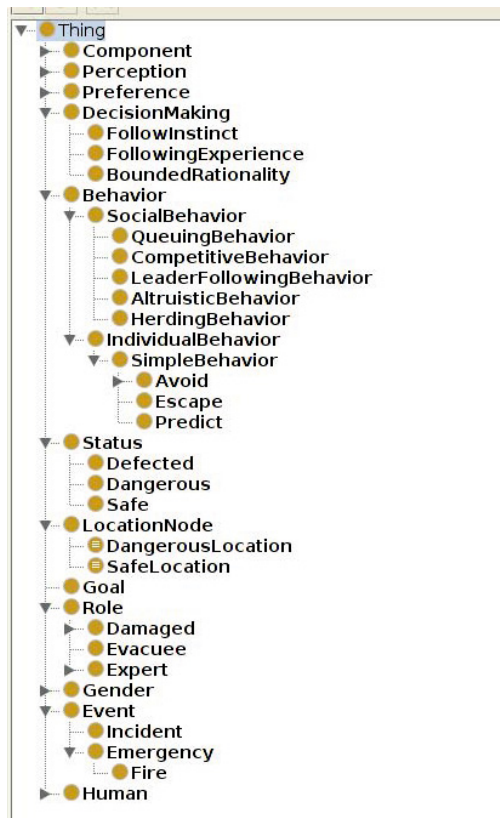

Fig. 2. The overview of ontology design in this research

an agent $A$ move from room $R_{a}$ to room $R_{b}$ with time $T_{1}$. Rooms and entire building elements are represented in node-based representation [18. The building elements such as rooms, corridors and exits, are considered and modeled on their physical accessibility. For example, room $R_{a}$ physically connects to room $R_{b}$ as showed in Fig 4, In this simulation, each agent has the same behavior and decision making process. Behavior model is described by an ontology which is comfortable to change when we want to edit the behavior model.

Simulation Scenario. This scenario aims to find the impact of accessibility of information toward human behavior changes. In the simulation, an evacuee is represented as an agent in simulation system. The agent assumed to be able to make a decision based on information it has at that time to respond the emergency situation. To find out the behavior changes impacted by information accessibility, this research observes the different outcomes between two simulation set. The first simulation set represents behaviors of evacuees whom can access necessary and updated information. For example, knowing of location of emergency exits and how the fire development is. The second simulation set is ones who can access limited and/or outdated of information. For example, knowing only some nor completely no idea of emergency exit locations, and knowing of an outdated of fire development in twenty minutes ago. 


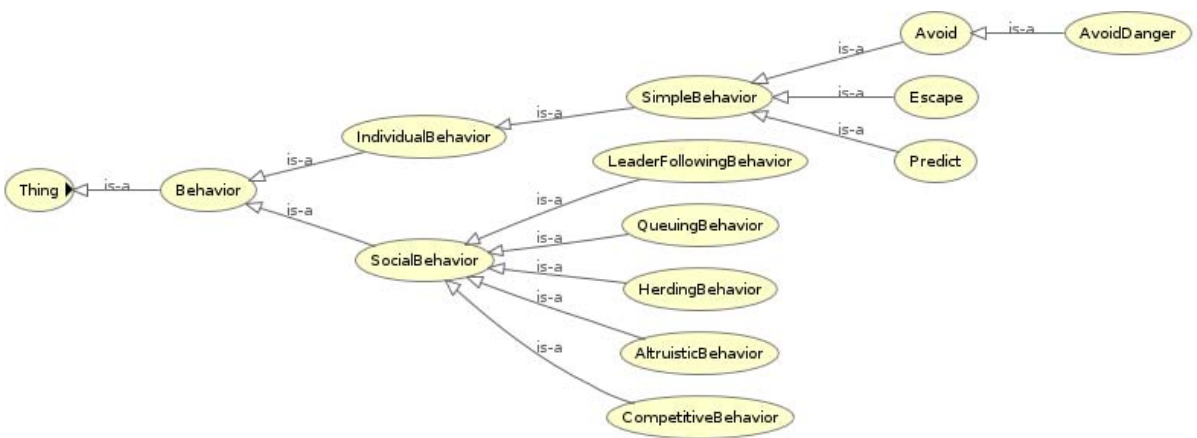

Fig. 3. Example of ontology design describing behavior concept in this simulation framework. However, in this research we focus on only IndividualBehavior.
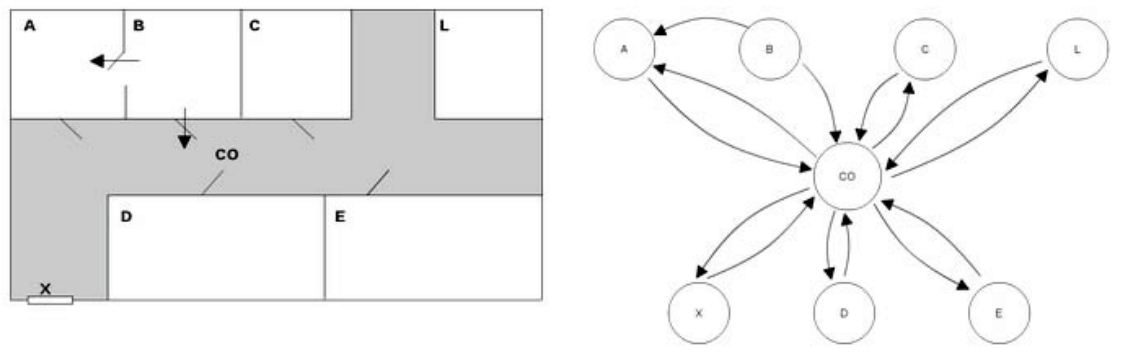

Fig. 4. The building's elements are modeled based on their accessibility 18 . A node represents to an element of building. For example, a floor plan has rooms $A, B, C, D, E$, elevator $L$, corridor $C O$ and an exit $X$ which are represented their physical accessibility graph.

Simulation Parameters. In the real emergency situation, there are many factors that is hard to be predicted or calculated in advance. For example, fire development's speed, processing time for an individual to take for a decision making. We design these factors as adjustable parameters in the simulation. Some parameters are described as the following list below.

- time limit - exceeded time means the building is too dangerous to survive. In that case, remaining individuals who got stuck in the building are fail to escape the building fire.

- individuals information processing time - each individual requires a period of time to process information they have to make a decision. The more information an individual perceive, the more processing time it take.

- fire development - it is a speed of fire spreading from one location to neighbor locations. All fire in the building have the same speed. 
Simulation Rules. This research attempts to explain complex phenomena using small and simple simulation parts. Each agent in the simulation shares common characters.

- an agent makes a decision right it gets updated information - As being explained in previous section, the simulation is divided into two groups. The first group can access rich and fresh information. The second group can access limited and outdated information. When agents in each group perceive information which controlled by Information Accessibility Controller module as shown in Fig, 1 they start to make a decision for the next move based on the given information.

- making a decision by the most favourable option - The decision making process will scan for all possible options an individual has, then predict the consequence for each option, and finally select the most favourable option. The criteria to make a decision is described in the ontology. For example, to avoid dangerous places and select the shortest path to the nearest safe exit.

\section{Discussion and Future Work}

This research proposes an ontology based simulation framework to study how situation awareness impact to human behavior in building fire environment. Even though this research is rely on assumptions that occupants have rational reaction in emergency and their decision making is based on simple rules which the result might be doubted whether it can reflexes the behavior in real situation. However, this is a good start of an ontology based simulation framework for studying human behavior changes impacted by information accessibility in an indoor emergency. In the near future, we can improve the rules to handle with more complex condition. Using ontology also has advantage of reuse and sharing knowledge from experts or other existing research studies. These make this research more flexible to adapt with different study purposes. For example, studying other information affecting human behavior, what will happen if occupants have some preference such as prefer to help other impaired occupants and evacuate the building together. These issues can be done by changing ontology and simulation rules. Moreover, the more understanding of relationship between human behavior and information accessibility could improve the indoor sensing technology in the near future. For example, under the critical situation which each individual could not process all information with the limited time. This knowledge might help us to prioritize which typ of information is the vital information to escape the emergency.

\section{References}

1. Hasofer, A.M., Thomas, I.: Analysis of fatalities and injuries in building fire statistics. Fire Safety Journal 41(1), 2-14 (2006)

2. Ouellette, M.: Visibility of exit signs. Progressive Architecture 74(7), 39-42 (1993) 
3. Kobes, M., Helsloot, I., de Vries, B., Post, J.G., Oberijé, N., Groenewegen, K.: Way finding during fire evacuation; An analysis of unannounced fire drills in a hotel at night. Building and Environment 45(3), 537-548 (2010)

4. Kobes, M., Helsloot, I., de Vries, B., Post, J.G.: Building safety and human behaviour in fire: A literature review. Fire Safety Journal 45(1), 1-11 (2010)

5. Fahy, R.F., Proulx, G.: Human behavior in the world trade center evacuation. In: Fire Safety Science-Proceedings of the Fifth International Symposium, pp. 713-724 (1998)

6. Fahy, R.F., Proulx, G., Aiman, L.: Panic or not in fire: Clarifying the misconception. Fire and Materials 36(5-6), 328-338 (2012)

7. Manoj, B.S., Baker, A.H.: Communication challenges in emergency response. Communications of the ACM 50(3), 51-53 (2007)

8. Vanderford, M.L., Nastoff, T., Telfer, J.L., Bonzo, S.E.: Emergency communication challenges in response to hurricane katrina: Lessons from the centers for disease control and prevention. Journal of Applied Communication Research 35(1), 9-25 (2007)

9. Joe Wilson, F.S.: Addressing diverse human behavior issues in the new era of multilayered emergency communications. Copyright: 9-1-1 Magazine, Feature Content, http: //www . federalsignal-indust . com/news/addressing-diverse-humanbehavior-issues-new-era-multi-layered-emergency-communications

10. Grosshandler, W.L., Bryner, N., Madrzykowski, D., Kuntz, K.: Report of the technical investigation of the station nightclub fire. National Institute of Standards and Technology Gaithersburg, MD (2005)

11. Batson, C.D., Powell, A.A.: Altruism and prosocial behavior. In: Handbook of Psychology (2003)

12. Pan, X.: Computational modeling of human and social behaviors for emergency egress analysis. PhD thesis, Stanford University (2006)

13. Noy, N.F., McGuinness, D.L., et al.: Ontology development 101: A guide to creating your first ontology (2001)

14. Helbing, D., Farkas, I., Vicsek, T.: Simulating dynamical features of escape panic. Nature 407(6803), 487-490 (2000)

15. Still, G.K.: Crowd dynamics. PhD thesis, University of Warwick (2000)

16. Low, D.J.: Statistical physics: Following the crowd. Nature 407(6803), 465-466 (2000)

17. Ketchell, N., Holt, A., Kinsella, K.: A technical summary of the aea egress code. tech. rep., Tech. Rep. 1, AEA Technology (2002)

18. Damrongrat, C., Kanai, H., Ikeda, M.: Increasing situational awareness of indoor emergency simulation using multilayered ontology-based floor plan representation. In: Yamamoto, S. (ed.) HIMI/HCII 2013, Part II. LNCS, vol. 8017, pp. 39-45. Springer, Heidelberg (2013) 